\title{
S100A4 promotes endometrial cancer progress through epithelial-mesenchymal transition regulation
}

\author{
TENG HUA, SHUANGGE LIU, XIAOYAN XIN, LIQIONG CAI, RUI SHI, \\ SHUQI CHI, DILU FENG and HONGBO WANG
}

\begin{abstract}
Department of Obstetrics and Gynecology, Union Hospital, Tongji Medical College, Huazhong University of Science and Technology, Wuhan, Hubei 430022, P.R. China
\end{abstract}

Received January 6, 2016; Accepted February 22, 2016

DOI: $10.3892 / o r .2016 .4760$

\begin{abstract}
Epithelial-mesenchymal transition (EMT) is a major cause of endometrial cancer (EC) to initiate invasion and metastasis. S100A4, a calcium-binding protein, is implicated in multistage of tumorigenesis and tumor progression. The correlation between S100A4 and EMT in EC is still unclear. This study was aimed to clarify the role of S100A4 in EC and the relationship between S100A4 expression and EMT markers. S100A4, E-cadherin, and vimentin were detected in tissues of EC patients $(n=50)$ by immunohistochemistry. The impact of S100A4 on EC cell proliferation, migration and invasion was investigated via RNA interference, and the correlation between S100A4 and EMT markers were also explored. The results showed that S100A4 was significantly increased in epithelial cells of EC compared with the normal endometrium $(\mathrm{P}<0.05)$, also S100A4 level was positively related to age $(\mathrm{P}=0.021)$, histological grade $(\mathrm{P}<0.001)$, and lymph node metastasis $(\mathrm{P}<0.001)$. Additionally, silencing of S100A4 remarkably attenuated EC cell migration and invasion. Significant morphological change accompanied with the downregulation of EMT markers, E-cadherin and vimentin were also observed. Aberrant S100A4 expression may predict EC progression and play an important role in regulating EC cell invasion through EMT regulation. Hence, S100A4 is a promising therapeutic target.
\end{abstract}

\section{Introduction}

Endometrial cancer (EC), the most common type of female genital tract malignancy, with substantial cancer related

Correspondence to: Dr Hongbo Wang or Dr Dilu Feng, Department of Obstetrics and Gynecology, Union Hospital, Tongji Medical College, Huazhong University of Science and Technology, 1277 Han Kou Jie Fang Road, Wuhan, Hubei 430022, P.R. China

E-mail: hb_wang1969@sina.com

E-mail: fengdilu@126.com

Key words: endometrial cancer, S100A4, epithelial-mesenchymal transition, invasion mortality. It was estimated that there were 52,630 new cases of EC with 8,590 deaths in 2014 (1). Approximately 70\% of EC patients are histologically adenocarcinomas, which may arise from complex atypical hyperplasia and closely link to estrogen overdose, metabolic disturbance and positive hormone receptors $(2,3)$. The prognosis of EC patients is affected by several risk factors, including tumor grade, myometrial invasion, lymphovascular space invasion, non-endometrioid histology and cervical stromal involvement (3). Metastasis is a major cause of mortality and recurrence in endometrial adenocarcinoma patients. In the process of tumor metastasis, numerous adhesion molecules, cytoskeleton interacting proteins, extracellular matrix proteins and angiogenesis related proteins are involved $(4,5)$. Further elucidating the mechanisms of tumor aggressiveness is meaningful for endometrial cancer patients to acquire better progression-free survival (PFS) and overall survival (OS) chance.

The S100 calcium-binding protein family has been shown to play an essential role in multiple steps of tumorigenesis and aggressiveness (6). Frequent locus gene diversification and protein conformational alteration facilitate their wide range of functions both intracellular and extracellular, such as proliferation, apoptosis, invasion, $\mathrm{Ca}^{2+}$ homeostasis and energy metabolism (7). Accumulating evidence suggest that S100A4, a member of S100 family, was overexpressed in various malignancies. Its pivotal role in promoting tumor progression and metastasis made it a valid predictor for poor outcome (8-11). Additionally, therapeutic targeting of S100A4 significantly inhibit tumor progress, indicating its potential value in human cancer treatment (12). The evidence for expression and functions of S100A4 in EC remains scant. Accordingly, S100A4 was overexpressed in high-grade EC which heralded poor prognosis $(13,14)$. However, little is known about the exact mechanisms of S100A4 in EC progression.

Epithelial-mesenchymal transition (EMT) has been demonstrated to be implicated in tumor progression and metastasis. During this process epithelial cells convert to a more migratory and invasive phenotype, which is accompanied with downregulated expression of epithelial markers, particularly E-cadherin, and induced expression of mesenchymal markers (15-17). Numerous studies indicated that S100A4 involved in EMT process and is considered as a key EMT molecular marker (18-20). Although the molecular 
mechanisms causing EMT has been well established in recent years, the implication of EMT in endometrial cancer pathogenesis and whether S100A4 is involved remains to be investigated.

In the present study, augmented cytoplasmic expression of S100A4 in poorly-differentiated EC was observed which closely related to EMT markers. We hypothesized that S100A4 promote EC metastasis via modulating EMT process, mainly affecting E-cadherin and vimentin expression. To confirm this, the role of S100A4 in HEC-1B cell migration, invasion, and morphological transition was studied, and the impact of S100A4 on EMT marker expression was investigated.

\section{Materials and methods}

Patients and samples. Formalin-fixed paraffin-embedded specimens were obtained from patients diagnosed with EC $(n=50)$ and atypical endometrial hyperplasia $(n=20)$ and who underwent surgical resection at Wuhan Union Hospital from 2010 to 2013. Those administered prior chemotherapy, hormone therapy or radiotherapy were excluded. The Surgical staging (I-IV) and histological grades (G1-G3) were established based on the criteria of the International Federation of Gynecology and Obstetrics (FIGO) for EC. The clinicopathological characteristics, such as age, pathological grading, stage, lymph node metastasis and myometrial invasion depth, were also collected. The present study was approved by The Ethics Committee of Tongji Medical College, Huazhong University of Science and Technology (IORG no: IORG0003571) after receiving informed consent from the patients.

Immunohistochemistry (IHC) and assessments. The four micrometer-thick tissue slides were deparaffinized with xylene and rehydrated in graded alcohol. Slides were then immersed in $0.01 \mathrm{M}$ sodium citrate buffer ( $\mathrm{pH} \mathrm{6.0)}$ and heated on microwave for $20 \mathrm{~min}$. This was followed by endogenous peroxidase activity blocking with $3 \%$ hydrogen peroxide $(15 \mathrm{~min})$ and nonspecific staining blocking with $10 \%$ normal goat serum (30 min). Then $200 \mu \mathrm{l}$ of antibodies against S100A4 (1:800; CST, \#13018), vimentin (1:100, Santa Cruz Biotechnology (Santa Cruz, CA, USA), sc-7557), and E-cadherin (1:100, Santa Cruz Biotechnology, sc-7870) were added on each tissue slide and incubated in a humidified box at $4^{\circ} \mathrm{C}$ overnight. For negative control, we added phosphate-buffered saline (PBS) instead of the primary antibody. Slides were then treated with Envision/HRP, rabbit secondary antibodies for $30 \mathrm{~min}$. Diaminobenzidine (DAB) substrate was added on each slide for visualizing, followed by rinsing, and counterstaining with hematoxylin. Finally, the slides were dehydrated in 4 different concentrations of alcohol and cleared in xylene.

The intensity evaluation of IHC staining was performed independently by two pathologists. Positive S100A4 staining was analyzed according to cytoplasmic and/or nuclear staining of the tumor cells. The scoring categories take into account both the intensity of IHC color reaction (none, 0 ; weak, 1 ; moderate, 2 ; strong, 3 ) and the percentage of positive tumor cells $(<10 \%, 1 ; 10-49 \%, 2 ;>50 \%, 3)$. The final score was the sum of the two scores. Histological score $\leq 1$ point was defined as negative, whereas $\geq 2$ points were considered positive.
Cell culture and shRNA transfection. The human endometrial cancer cell lines HEC-1B, Ishikawa, and KLE were purchased from the American Type Culture Collection (ATCC), cultured in Dulbecco's modified Eagle's medium (DMEM, Hyclone, USA) containing $10 \%$ fetal bovine serum with $100 \mathrm{U} / \mathrm{ml}$ penicillin and $100 \mu \mathrm{g} / \mathrm{ml}$ streptomycin, at $37^{\circ} \mathrm{C}$ in a humidified atmosphere containing $5 \% \mathrm{CO}_{2}$.

shRNAs for S100A4 and vector was commercially purchased from GeneChem (Shanghai, China). The target sequence for synthetic oligonucleotide primers of S100A4 shRNA were: shS100A4-1: CAGATAAGCAGCCCAGGAA; shS100A4-2: CCATGATGTGTAACGAATT; shS100A4-3: TCCAAGAGTACTGTGTCTT; shS100A4-4: CTGCTTT CCAGAAGCTGAT; The target sequence: CTCCGAACG TGTCACGTT was used as a non-silencing control (vector). The vector plasmid and plasmid carrying S100A4 shRNA were transfected into HEC-1B cells using Lipofectamine 2000 (Invitrogen, Carlsbad, CA, USA) following manufacturer's recommendations. The cells were harvested after $48 \mathrm{~h}$ of transfection. Stably transfected clones of shS100A4-4 and vector HEC-1B cells were selected using $100 \mu \mathrm{g} / \mathrm{ml}$ ampicillin (Sigma, Santa Clara, CA, USA) for 3 days.

RNA isolation and quantitative real-time PCR (qRT-PCR). Total RNA was extracted from the transfected cells using the TRIzol (Invitrogen) and First-strand cDNA was reverse-transcribed from $2 \mu \mathrm{g}$ total RNA using the Prime Script RT reagent kit (Takara, Kyoto, Japan) according to the manufacturer's protocol. cDNA samples $(2 \mu \mathrm{l})$ were added to quantitative PCR in $20 \mu 1$ reactions using SYBR Premix Ex Taq (Takara). The primers involved were: h-S100A4 (251 bp): 5'-TACT CGGGCAAAGAGGGTGA-3' (sense) and 5'-CATTTCTT CCTGGGCTGCTTA-3' (antisense). h-GAPDH (255 bp): 5'-ACTTTGGTATCGTGGAAGGACTAT-3' (sense) and 5'-GTTTTTCTAGACGGCAGGTCAGG-3' (antisense). Values on the $\mathrm{y}$-axis represent $2^{(-\Delta \Delta C t)}$, while $\Delta \mathrm{Ct}$ is the difference between target-gene $\mathrm{Ct}$ and GAPDH Ct.

Western blot analysis. The transfected HEC-1B cells were rinsed in ice-cold PBS twice and lysed in lysis buffer. The protein concentration was measured using the Bio-Rad assay system. Proteins $(20 \mu \mathrm{g})$ were boiled for $10 \mathrm{~min}$ and run on $15 \%$ SDS-PAGE gels. Then transferred onto nitrocellulose membrane and the membranes were blocked with $5 \%$ skim milk in $1 \mathrm{X}$ TBS buffer containing $0.1 \%$ Tween- 20 and then probed with antibodies against S100A4 (1:2000, CST, \#13018), GAPDH (1:800, Santa Cruz Biotechnology, sc-25778), vimentin (1:1000, Santa Cruz Biotechnology, sc-7557), E-cadherin (1:1000, Santa Cruz Biotechnology, sc-7870) at $4^{\circ} \mathrm{C}$; Horseradish peroxidase (HRP)-conjugated anti-rabbit IgG was used as the secondary antibody. The bands were detected by enhanced Chemiluminescence reagents (Thermo Fisher Scientific, Inc., Waltham, MA, USA). The optical density was quantified using Image $\mathrm{Lab}^{\mathrm{TM}}$ analysis software. The relative expression data have been normalized by GAPDH expression.

Immunofluorescence staining. Cells were cultivated in six-well plates overnight. Then washed by PBS twice and fixed with $4 \%$ paraformaldehyde for $15 \mathrm{~min}$. Cells for vimentin and tubulin 

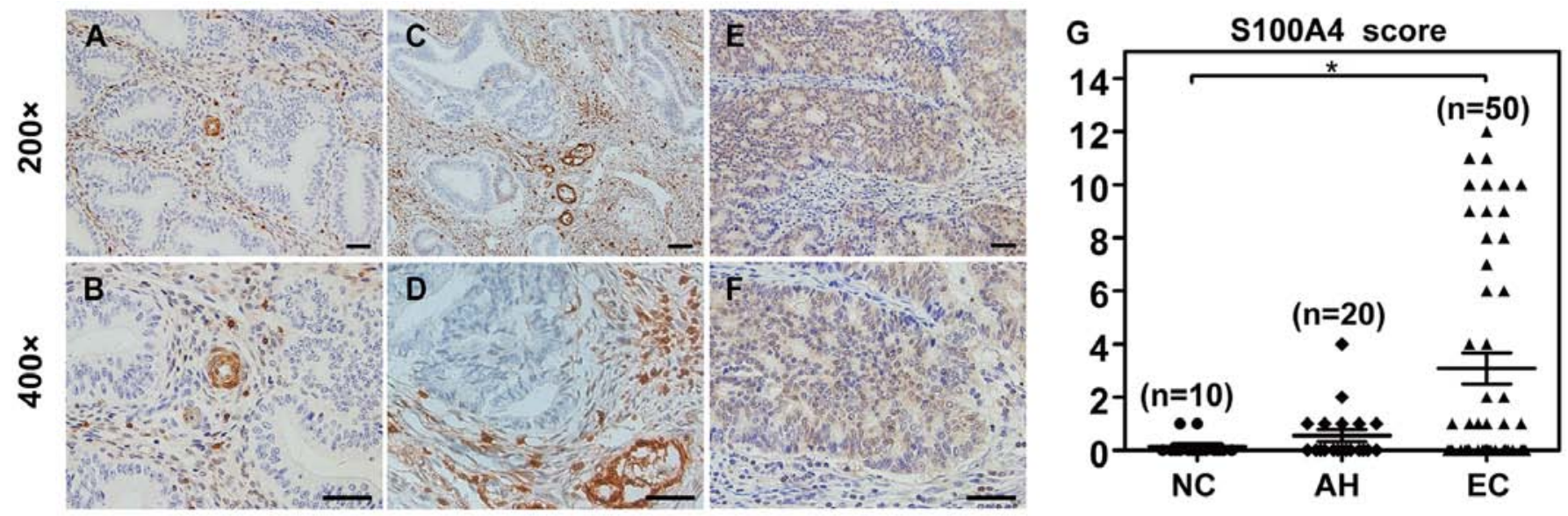

Figure 1. S100A4 protein was overexpressed in the cytoplasm of cancer cells in EC. Representative images of immunohistochemical assay show S100A4 protein (brown) in normal endometrium (A and B), atypical hyperplasia of endometrium (C and D), endometrial cancer (E and F). Scale bars: $20 \mu \mathrm{m}$. (G) Histological scores of S100A4 in patients of normal (NC), atypical hyperplasia of endometrium $(\mathrm{AH})$, and endometrial cancer $(\mathrm{EC})$. * $\mathrm{P}<0.05$.

staining were treated with $0.1 \%$ Triton $\mathrm{X}-100$ for enhancing permeability and blocked with $5 \%$ BSA for $1 \mathrm{~h}$, then incubated with primary antibodies overnight. After two washes with PBS, incubated with Cy3-conjugated goat anti-mouse or anti-rabbit IgG antibody (1:100 dilution, Guge, Wuhan, China) in the dark for $1 \mathrm{~h}$, then counterstained with DAPI. Images were taken using an Olympus Fluoview IX-300 laser-scanning confocal microscope.

CCK- 8 assay. The CCK-8 assay was adopted to detect the effect of S100A4 in EC cell viability. Briefly, the shS100A4-4 and control group HEC-1B cells were plated in a 96-well plate at the density of $5 \times 10^{3} / 100 \mu 1$ per well. Cell growth was analyzed every $24 \mathrm{~h}$ using a CCK-8 commercial kit (SigmaAldrich) following manufacturer's instructions.

Wound-healing assay. The shS100A4-4 and control group HEC-1B cells were plated in a six-well plate, $1 \times 10^{5}$ per well. When cells were grown to confluence as a monolayer, a wound was created using a sterile $200 \mu \mathrm{l}$ pipette tip followed with serum starvation for $48 \mathrm{~h}$. Photographs of the wounded area were taken at the time of wounding and every $12 \mathrm{~h}$ afterwards for 2 days to determine the rate of wound closure. The migration percentage was calculated using the following formula: $[\Delta$ area/area $(0 \mathrm{~h})] \times 100 \%$.

Transwell invasion assay. The shS100A4-4 and control group cells were plated in the upper chamber coated with Matrigel basement membrane matrix (BD Biosciences, Bedford, MA, USA) in serum-free medium, $5 \times 10^{4}$ cells per well. A chemoattractant ( $10 \%$ fetal bovine serum) was added to the lower chamber and incubated for $48 \mathrm{~h}$. Invasive cells, were fixed in $4 \%$ paraformaldehyde and stained in $0.5 \%$ crystal violet, were counted in five random fields of each trans-well filter using a microscope.

Statistical analyses. Statistical analysis was performed using the SPSS software 21.0 statistical software (Chicago, IL, USA). Chi-square test and Fisher's exact tests were used when comparing frequencies between groups. Values represent the mean $\pm \mathrm{SD}$ of one representative experiment from a series of independent experiments. Data were analyzed using the Student's t-test. P-values $<0.05$ were statistically significant.

\section{Results}

S100A4 is overexpressed in the cytoplasm of cancer cells in $E C$. Immunohistochemistry was performed to characterize the quantity and localization of S100A4 protein. In all normal endometrial tissues (10/10) and the majority of atypical endometrial hyperplasia tissues $(18 / 20)$, the expression of S100A4 was confined in the mesenchymal sections, particularly in endometrial stromal cells and vascular structures. None of the glandular epithelial cells expressed S100A4 protein (Fig. 1A-D). In contrast, 32\% (16/50) of the endometrial cancer tissues overexpressed S100A4 both in mesenchymal sections and tumor epithelial cells (Fig. 1E and F). Statistically, cytoplasmic expression of S100A4 protein in endometrial cancer epithelial cells was significantly higher than normal group ( $\mathrm{P}=0.014)$. However, there was no difference between endometrial cancer and atypical hyperplasia endometrium $(\mathrm{P}=0.057)$ (Fig. 1G).

Positive cytoplasmic S100A4 protein indicates EC progression. To investigate the clinical significance of S100A4 in EC patients, correlation between cytoplasmic S100A4 protein expression and clinicopathological parameters was analyzed. Positive staining for S100A4 in tumor cells was significantly associated with cell differentiation, more frequent incidence was observed in high-grade cases (G2/G3, 14/24) compared with the low-grade cases (G1,2/26). Age and lymph node metastasis also showed remarkable correlation with expression of S100A4. No significant correlation between S100A4 expression and FIGO stage or myometrial invasion depth was found, though a related trend was observed (Table I).

S100A4 silencing suppresses migration and invasion capability of HEC-1B cells. The high invasive EC cell line HEC-1B possessed high endogenous S100A4 when compared to low invasive EC cell lines Ishikawa and KLE 
A

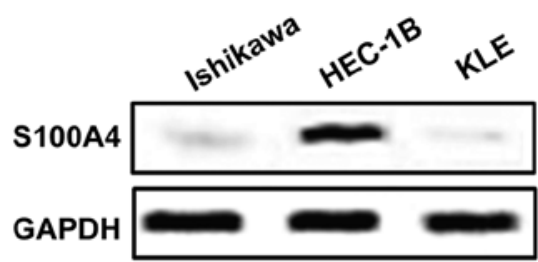

B

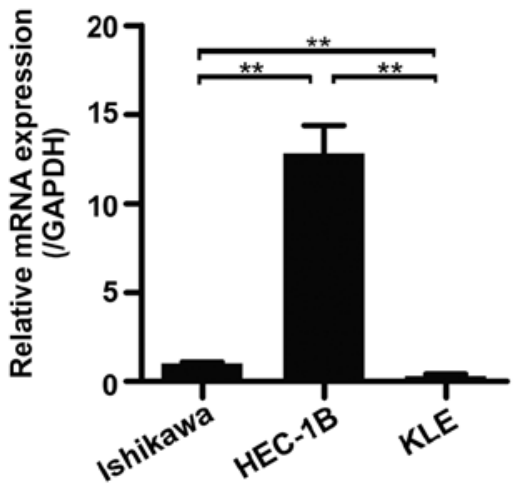

C

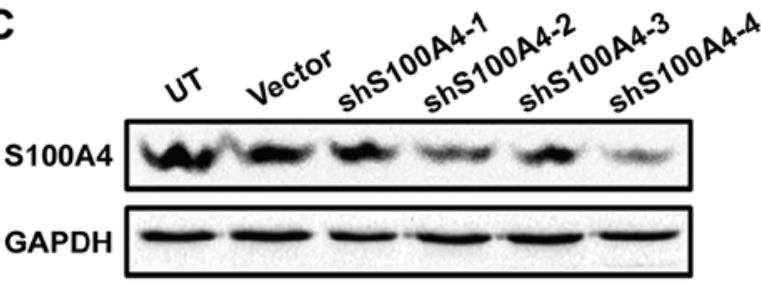

D

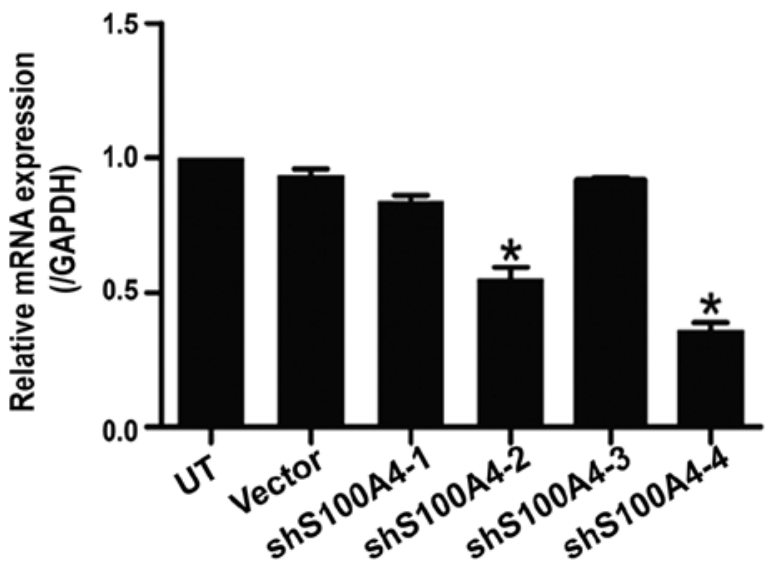

Figure 2. Expression of S100A4 in EC cell lines and shRNA silenced S100A4 expression in HEC-1B cells. (A and B) Western blot and qRT-PCR analysis of S100A4 protein and mRNA levels in EC cell lines (Ishikawa, HEC-1B, KLE). (C and D) Western blot and qRT-PCR analysis of S100A4 demonstrating the transfection efficiency. GAPDH was used as internal control. ${ }^{*} \mathrm{P}<0.05,{ }^{* *} \mathrm{P}<0.01$.

(Fig. 2A and B). To study the effects of S100A4 on migration and invasion of HEC-1B cells, four shRNAs were constructed and transfected into HEC-1B cells. Transient transfection of all the four groups, except for shS100A4-3 group, exhibited an inhibitory effect on S100A4 expression in HEC-1B cells (Fig. 2C and D). The shS100A4-4 group provided the strongest efficacy. Further, the inhibitory effect in shS100A4-4 group was maximal after stable screening with puromycin confirmed by western blotting (Fig. 4C). Functional experiments demonstrated that the migration and invasion of HEC-1B cells were significantly suppressed in shS100A4-4 group compared with control group (Fig. 3B and $\mathrm{C}$ ). However, no differences were detected in viability of HEC-1B cells (Fig. 3A). Of note, marked morphological change was also observed in shS100A4-4 group with attenuated EMT-like phenotype.

Downregulation of $S 100 A 4$ reverses EMT. To study the effects of S100A4 on EC cell cytoskeleton, optical microscope observation and immunofluorescence staining against tubulin were utilized. We found morphological changes and cytoskeleton reorganization when S100A4 was downregulated (Fig. 4A and B). The HEC-1B cells lost their spindle-shaped morphology and showed suppressed spreading. Besides, E-cadherin and vimentin, which account for major epithelial and mesenchymal markers were also detected in shS100A4-4 cells and EC specimens. E-cadherin was mainly expressed in the membrane whereas vimentin mostly in the cytosol. In addition, concurrent upregulation of E-cadherin and downregulation of vimentin upon ablation of S100A4 were observed (Fig. 4D) and further confirmed by western blotting (Fig. 4C). Moreover, in high-grade EC samples, high S100A4 expression closely correlated with
Table I. Clinicopathological characteristics and cytoplasmic S100A4 expression in patients with endometrial cancer.

\begin{tabular}{lcccc}
\hline & \multicolumn{3}{c}{ S100A4 expression } & \\
\cline { 2 - 4 } Parameters & $(\mathrm{n}=50)$ & $+(\%)$ & $-(\%)$ & P-value \\
\hline Age & & & & \\
$\quad<50$ & 14 & $1(7.1)$ & $13(92.9)$ & 0.021 \\
$\geq 50$ & 36 & $15(41.7)$ & $21(58.3)$ & \\
FIGO stage & & & & \\
I/II & 39 & $10(25.6)$ & $29(74.4)$ & 0.147 \\
III/IV & 11 & $6(54.5)$ & $5(45.5)$ & \\
Histologic grade & & & & \\
G1 & 26 & $2(7.7)$ & $24(92.3)$ & $<0.001$ \\
G2/G3 & 24 & $14(58.3)$ & $10(41.7)$ & \\
Myometrial invasion & & & & \\
$<1 / 2$ of myometrium & 34 & $8(23.5)$ & $26(76.5)$ & 0.061 \\
$\geq 1 / 2$ of myometrium & 16 & $8(50)$ & $8(50)$ & \\
LN metastasis & & & & \\
Absent & 44 & $2(4.5)$ & $42(95.5)$ & $<0.001$ \\
Present & 6 & $4(66.7)$ & $2(33.3)$ & \\
\hline
\end{tabular}

FIGO, International Federation of Gynecology and Obstetrics.

decreased E-cadherin and elevated vimentin expression (Fig. 4E). These results suggested a crucial role for S100A4 in EMT-mediated metastasis of EC. 
A

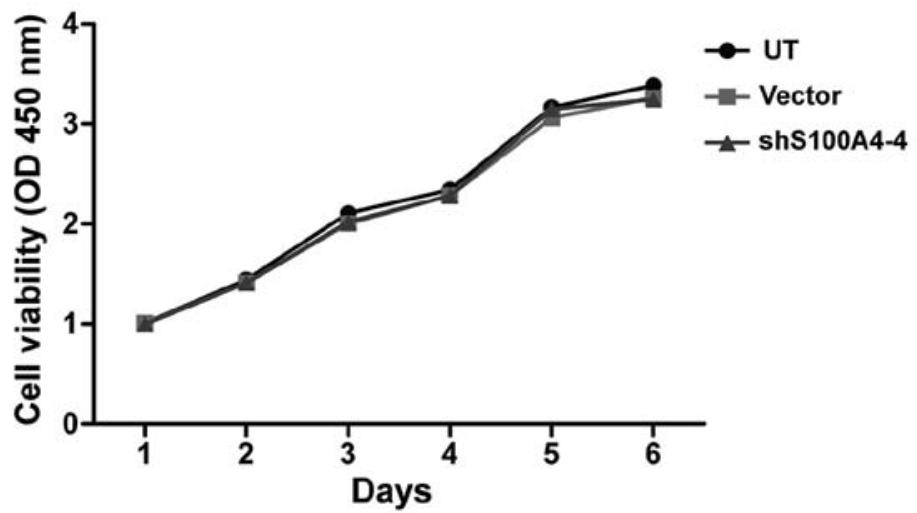

B

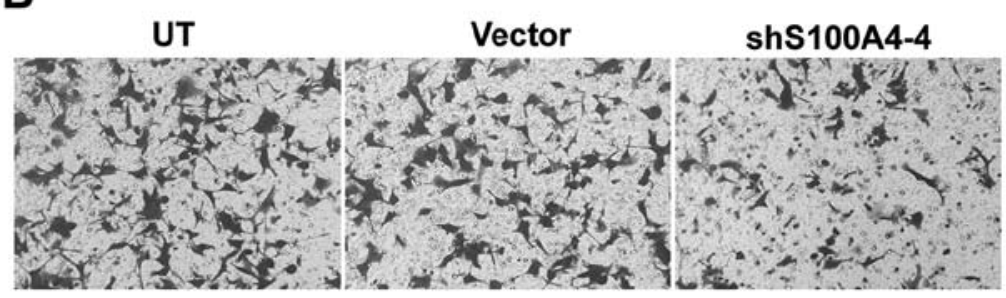

C UT Vector shS100A4-4

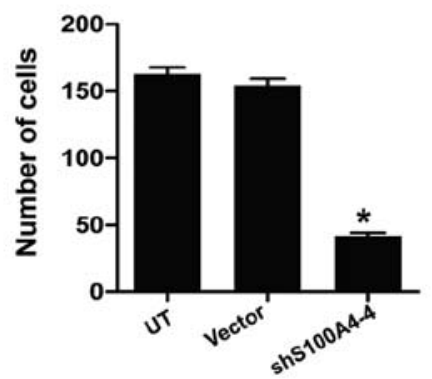

oh

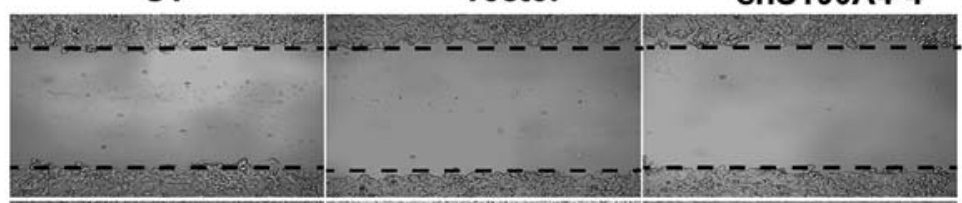

$24 \mathrm{~h}$
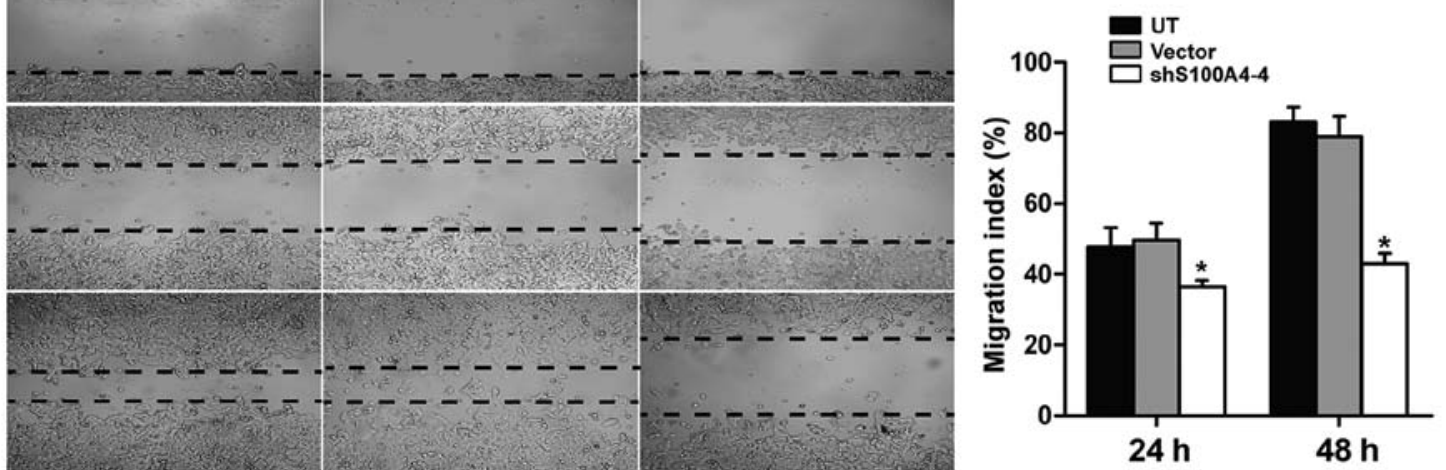

Figure 3. Blocking S100A4 suppressed migration and invasion capability of HEC-1B cells. (A) CCK-8 assay showed S100A4 has no impact on HEC-1B cell proliferation. (B) Invasive property of HEC-1B cells induced by S100A4 was analyzed by Transwell invasion assay (x200). (C) HEC-1B cell migration ability was measured by testing the index of wound closure at $0,24,48 \mathrm{~h}(\mathrm{x} 200)$. ${ }^{*} \mathrm{P}<0.05$.

\section{Discussion}

Elevated S100A4 protein expression has been detected in endometrial carcinoma and other tumors, and higher S100A4 protein levels concordant with advanced stages of cancer $(13,21)$. Thus, a positive correlation was established between upregulation of S100A4 and tumor malignancy progression. In agreement with previous study, S100A4 was abundantly expressed in higher grade EC patients (14). Besides, a transitional expression pattern of S100A4 was observed in which S100A4 was undetectable in epithelial cells of lowgrade EC, normal endometrium and atypical endometrial hyperplasia while widely expressed in mesenchymal origins, especially in stromal cells and vascular structures. This kind of expression pattern was also found in prostate cancer which may imply an epithelial to mesenchymal transition (EMT) derived from gene hypomethylation (22). Since EMT has been recognized as a core molecular mechanism underlying cancer invasion and metastasis, the role of S100A4 was focussed on in EC progression. Additionally, S100A4 has also been shown to possess the ability of activating or integrating pathways to promote migratory phenotype, angiogenesis, extracellular matrix components (ECM) remodeling process (23-25). The crucial role of S100A4 in EC cell invasion and metastasis were confirmed by in vitro studies which elucidated that overexpression of S100A4 was associated with metastatic properties while targeting knockdown of S100A4 markedly reduced the migratory ability.

The clinical significance of S100A4 in cancer is still controversial. In cases of cancers, S100A4 was universally overexpressed and the excessive expression of S100A4 was an independent predictor for tumor progression, metastasis, and poor prognosis. For example, increased S100A4 expression was detected in the metastatic sections of breast cancer (26). Enhanced expression of S100A4 underlies lymph node metastasis and predicts recurrence in colorectal cancer (27). 
A

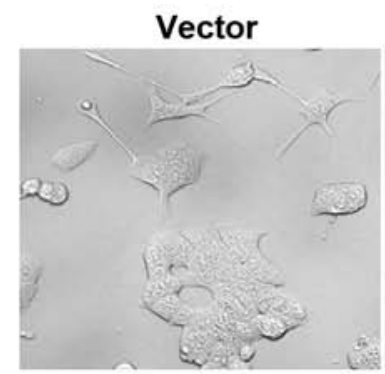

shS100A4-4

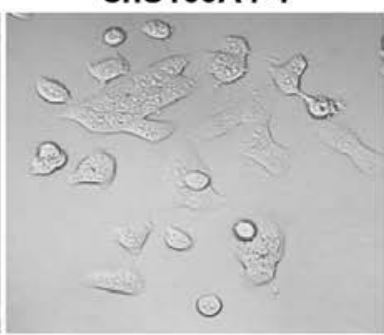

B

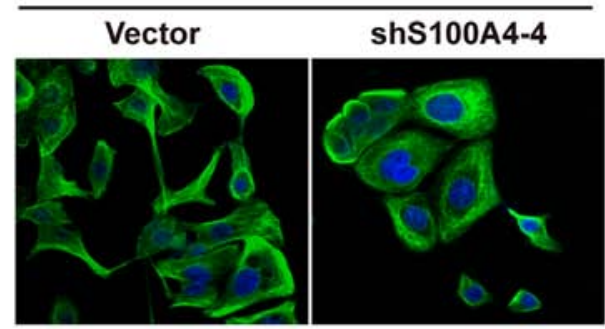

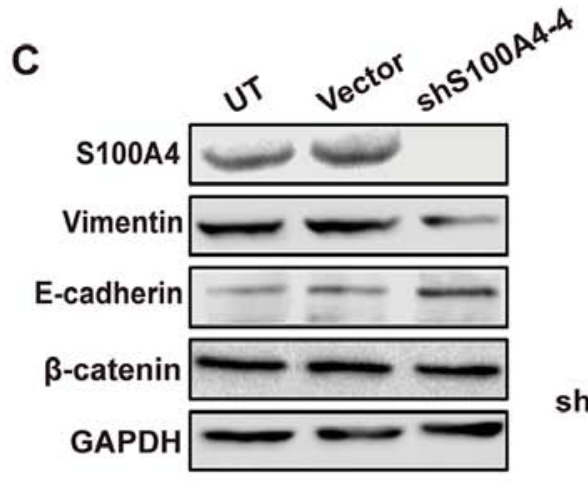

D
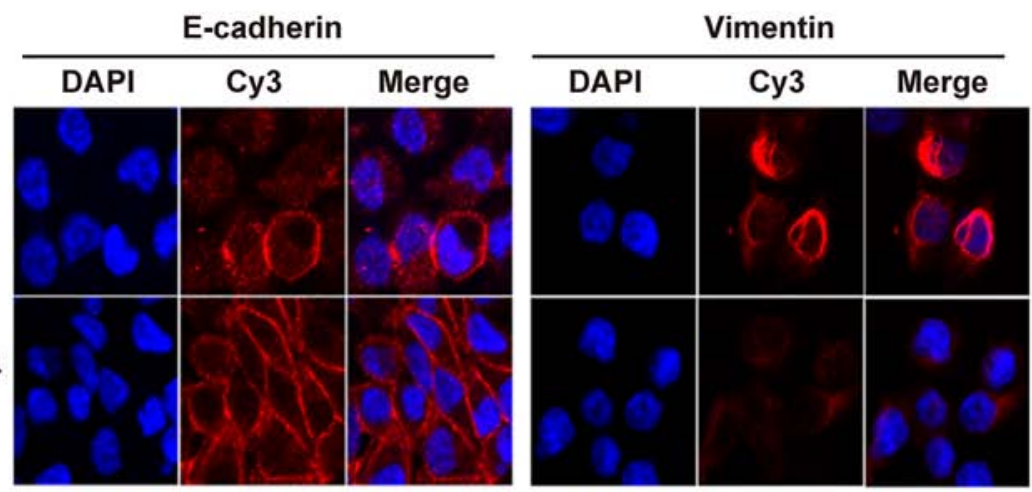

E Normal G1

G2

G3
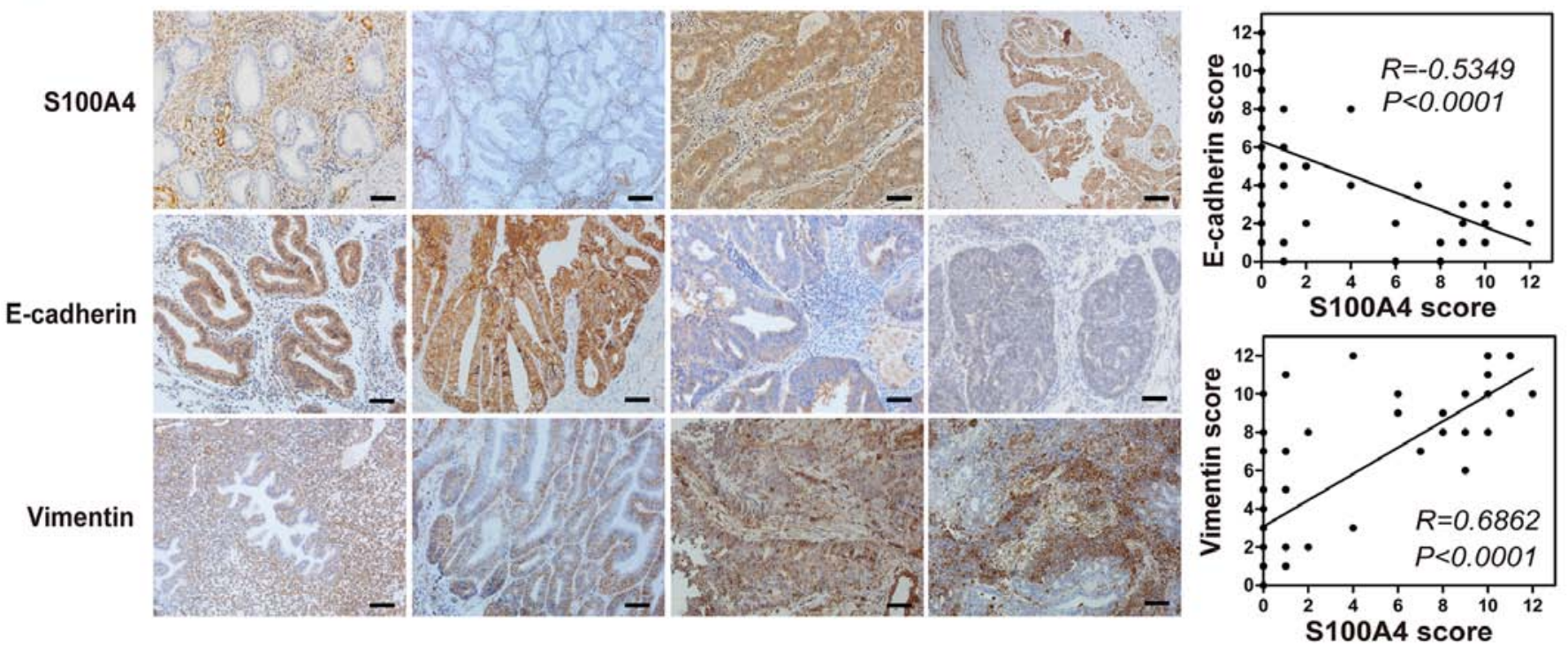

Figure 4. Downregulation of S100A4 reverses EMT. (A) S100A4 silencing reversed epithelial morphology in HEC-1B cells (x200). (B) Cytoskeleton changes of HEC-1B cells were determined by staining with tubulin (green), the nucleus were stained with DAPI (blue) and visualized by confocal microscopy (x600). (C) Expression levels of S100A4 and EMT markers were analyzed by western blotting. GAPDH was used as the internal control. (D) Immunofluorescence assay of EMT markers (red), the nucleus were stained with DAPI (blue) and visualized by confocal microscopy (x600). (E) Immunohistochemical assay of S100A4 and EMT markers (brown) in EC tissues with different pathological grades (x200). Scale bars: $50 \mu \mathrm{m}$.

However, some studies obtained contradictive results (28). Even so, the majority of the investigators believed that S100A4 indeed closely related to some clinical and pathological parameters. In the study of Chong et al, partly consistent with us, indicated that cytoplasmic expression of S100A4 was significantly related to the clinicopathological parameters of EC patients, including FIGO stage, histological grade, loss of progesterone receptor (PR) and lymph node metastasis. Kaplan-Meier survival analysis suggested a significant correlation between cytoplasmic expression of S100A4 and poorer outcome (13). The present study also showed relevance between S100A4 expression and age. Age represents an important risk factor for EC occurrence. Most EC are diagnosed after menopause and older women are more likely to have poorly differentiated endometrioid histology and higher stage disease. These results led to a hypothesis that S100A4 may be an effective indicator for EC progression.

Clinical and experimental evidence indicate a critical role of EMT interrelated process in progression and metastasis of EC. EMT is a complex and stepwise phenomenon which is indispensable for morphogenesis in embryonic development and is reinitiated during cancer progression forming a more invasive phenotype. The molecular mechanisms underlying EMT can comprise multiple extracellular signals and numerous 
secreted soluble factors that ultimately activate different transcription factors and signaling pathways (15). Disassembly of cell-cell and cell-ECM junction together with downregulation of epithelial marker E-cadherin is recognized as hallmark of EMT. Simultaneously, progressive loss of E-cadherin expression is always coupled with augmented expression of non-epithelial cadherin, e.g. N-cadherin, cadherin-11 and acquired mesenchymal markers, such as fibronectin, smooth muscle actin, or vimentin (29). Changes of these molecules were also found in human EC, as a steady decrease of E-cadherin and increase of vimentin was observed in high grade EC specimens. These observations conformed to the findings of Yang et al (30).

S100A4 has been considered as a crucial EMT mediator since directly controlled by Wnt/ $\beta$-catenin signaling pathway (31). While the role of S100A4 in regulating EMT process in EC is still unclear. The findings of Xie et al revealed a pivotal role of S100A4 in mediating EC invasion and clarified the regulation effect of TGF- $\beta 1$ on S100A4 expression (32). TGF- $\beta 1$ pathways could mediate EMT in various epithelial cell types. Given the explicit role of E-cadherin and vimentin in EMT, the current study found direct correlation between expression of S100A4 and EMT markers in EC for the first time, S100A4 is an upstream regulator of E-cadherin and vimentin. EC cells transfected with an S100A4 inhibition vector showed increased cell-cell adhesion morphology, plus downregulation of E-cadherin and upregulation of vimentin. Besides, S100A4 induced E-cadherin expression from membranous to membrano-cytoplasmic, indicated its pivotal role in malignant transformation of EC cells through EMT regulation. EMT molecules are more than merely cementing substances but also regulate cell polarity, differentiation, migration and invasion. These cellular events may be mediated via their intimate connection to the actin cytoskeletal network (33). S100A4 has been demonstrated to interact with components of cytoskeleton, such as F-actin, non-muscle tropomyosin, and non-muscle myosin, further regulating the cytoskeletal dynamics and cell motility (34). Conformational changes of tubulin in transfected cells provided clues for this effect.

In conclusion, our study demonstrates that positive cytoplasmic S100A4 was observed in high-grade EC as compared with the negative counterparts. S100A4 promoted migration and invasion capacity of EC cells via EMT modification. Thus, S100A4 might be a potential predictor for EC patient progress and targeting S100A4 could be a promising therapeutic target for EC treatment.

\section{Acknowledgements}

This work was supported by Natural Science Foundation of Hubei Province, China (grant no. 2015CFB237).

\section{References}

1. Siegel R, Ma J, Zou Z and Jemal A: Cancer statistics, 2014. CA Cancer J Clin 64: 9-29, 2014.

2. Kandoth C, Schultz N, Cherniack AD, Akbani R, Liu Y, Shen H, Robertson AG, Pashtan I, Shen R, Benz CC, et al; Cancer Genome Atlas Research Network: Integrated genomic characterization of endometrial carcinoma. Nature 497: 67-73, 2013.
3. Amant F, Mirza MR, Koskas M and Creutzberg CL: Cancer of the corpus uteri. Int J Gynaecol Obstet 131 (Suppl 2): S96-S104, 2015.

4. Psaila B and Lyden D: The metastatic niche: Adapting the foreign soil. Nat Rev Cancer 9: 285-293, 2009.

5. Valastyan S and Weinberg RA: Tumor metastasis: Molecular insights and evolving paradigms. Cell 147: 275-292, 2011.

6. Chen H, Xu C, Jin Q and Liu Z: S100 protein family in human cancer. Am J Cancer Res 4: 89-115, 2014.

7. Donato R, Cannon BR, Sorci G, Riuzzi F, Hsu K, Weber DJ and Geczy CL: Functions of S100 proteins. Curr Mol Med 13: 24-57, 2013.

8. Lee SJ, Choi SY, Kim WJ, Ji M, Lee TG, Son BR, Yoon SM, Sung R, Lee EJ, Youn SJ, et al: Combined aberrant expression of E-cadherin and S100A4, but not $\beta$-catenin is associated with disease-free survival and overall survival in colorectal cancer patients. Diagn Pathol 8: 99, 2013.

9. Dahlmann M, Okhrimenko A, Marcinkowski P, Osterland M, Herrmann P, Smith J, Heizmann CW, Schlag PM and Stein U: RAGE mediates S100A4-induced cell motility via MAPK/ERK and hypoxia signaling and is a prognostic biomarker for human colorectal cancer metastasis. Oncotarget 5: 3220-3233, 2014.

10. Lee SH, Kim H, Hwang JH, Shin E, Lee HS, Hwang DW, Cho JY, Yoon YS, Han HS and Cha BH: CD24 and S100A4 expression in resectable pancreatic cancers with earlier disease recurrence and poor survival. Pancreas 43: 380-388, 2014.

11. Burock S, Herrmann P, Wendler I, Niederstrasser M, Wernecke KD and Stein U: Circulating metastasis associated in colon cancer 1 transcripts in gastric cancer patient plasma as diagnostic and prognostic biomarker. World J Gastroenterol 21: 333-341, 2015.

12. Hernández JL, Padilla L, Dakhel S, Coll T, Hervas R, Adan J, Masa M, Mitjans F, Martinez JM, Coma S, et al: Therapeutic targeting of tumor growth and angiogenesis with a novel antiS100A4 monoclonal antibody. PLoS One 8: e72480, 2013.

13. Chong HI, Lee JH, Yoon MS, Suh DS, Kim K, Kim JY and Choi KU: Prognostic value of cytoplasmic expression of S100A4 protein in endometrial carcinoma. Oncol Rep 31: 2701-2707, 2014.

14. Xie R, Loose DS, Shipley GL, Xie S, Bassett RL Jr and Broaddus RR: Hypomethylation-induced expression of S100A4 in endometrial carcinoma. Mod Pathol 20: 1045-1054, 2007.

15. Lamouille S, Xu J and Derynck R: Molecular mechanisms of epithelial-mesenchymal transition. Nat Rev Mol Cell Biol 15: 178-196, 2014.

16. Zheng $\mathrm{H}$ and Kang Y: Multilayer control of the EMT master regulators. Oncogene 33: 1755-1763, 2014.

17. Kidd ME, Shumaker DK and Ridge KM: The role of vimentin intermediate filaments in the progression of lung cancer. Am J Respir Cell Mol Biol 50: 1-6, 2014.

18. Wang H, Shi J, Luo Y, Liao Q, Niu Y, Zhang F, Shao Z, Ding Y and Zhao L: LIM and SH3 protein 1 induces TGF $\beta$-mediated epithelial-mesenchymal transition in human colorectal cancer by regulating S100A4 expression. Clin Cancer Res 20: 5835-5847, 2014.

19. Lo JF, Yu CC, Chiou SH, Huang CY, Jan CI, Lin SC, Liu CJ, $\mathrm{Hu}$ WY and Yu YH: The epithelial-mesenchymal transition mediator S100A4 maintains cancer-initiating cells in head and neck cancers. Cancer Res 71: 1912-1923, 2011.

20. Nitta T, Mitsuhashi T, Hatanaka Y, Miyamoto M, Oba K, Tsuchikawa T, Suzuki Y, Hatanaka KC, Hirano S and Matsuno Y: Prognostic significance of epithelial-mesenchymal transition-related markers in extrahepatic cholangiocarcinoma: Comprehensive immunohistochemical study using a tissue microarray. Br J Cancer 111: 1363-1372, 2014.

21. McKiernan E, McDermott EW, Evoy D, Crown J and Duffy MJ: The role of S100 genes in breast cancer progression. Tumour Biol 32: 441-450, 2011.

22. Rosty C, Ueki T, Argani P, Jansen M, Yeo CJ, Cameron JL, Hruban RH and Goggins M: Overexpression of S100A4 in pancreatic ductal adenocarcinomas is associated with poor differentiation and DNA hypomethylation. Am J Pathol 160: 45-50, 2002.

23. Buetti-Dinh A, Pivkin IV and Friedman R: S100A4 and its role in metastasis - computational integration of data on biological networks. Mol Biosyst 11: 2238-2246, 2015.

24. Miranda KJ, Loeser RF and Yammani RR: Sumoylation and nuclear translocation of S100A4 regulate IL-1beta-mediated production of matrix metalloproteinase-13. J Biol Chem 285: 31517-31524, 2010. 
25. O'Connell JT, Sugimoto H, Cooke VG, MacDonald BA, Mehta AI, LeBleu VS, Dewar R, Rocha RM, Brentani RR, Resnick MB, et al: VEGF-A and Tenascin-C produced by S100A4 ${ }^{+}$stromal cells are important for metastatic colonization. Proc Natl Acad Sci USA 108: 16002-16007, 2011.

26. Kim HM, Jung WH and Koo JS: Expression of cancer-associated fibroblast related proteins in metastatic breast cancer: An immunohistochemical analysis. J Transl Med 13: 222, 2015.

27. Kang YG, Jung CK, Lee A, Kang WK, Oh ST and Kang CS: Prognostic significance of S100A4 mRNA and protein expression in colorectal cancer. J Surg Oncol 105: 119-124, 2012.

28. Jung EA, Cho HD, Lee JH and Oh MH: Clinicopathological significance of S100A4 expression in non-small cell lung carcinomas. Korean J Pathol 44: 477-482, 2010.

29. Thiery JP and Sleeman JP: Complex networks orchestrate epithelial-mesenchymal transitions. Nat Rev Mol Cell Biol 7: 131-142, 2006.

30. Yang WN, Ai ZH, Wang J, Xu YL and Teng YC: Correlation between the overexpression of epidermal growth factor receptor and mesenchymal makers in endometrial carcinoma. J Gynecol Oncol 25: 36-42, 2014.
31. Sack U, Walther W, Scudiero D, Selby M, Aumann J, Lemos C, Fichtner I, Schlag PM, Shoemaker RH and Stein U: S100A4induced cell motility and metastasis is restricted by the Wnt $/ \beta$-catenin pathway inhibitor calcimycin in colon cancer cells. Mol Biol Cell 22: 3344-3354, 2011.

32. Xie R, Schlumbrecht MP, Shipley GL, Xie S, Bassett RL Jr and Broaddus RR: S100A4 mediates endometrial cancer invasion and is a target of TGF-betal signaling. Lab Invest 89: 937-947, 2009.

33. Knights AJ, Funnell AP, Crossley M and Pearson RC: Holding tight: Cell junctions and cancer spread. Trends Cancer Res 8: 61-69, 2012.

34. Goh Then Sin C, Hersch N, Rudland PS, Barraclough R, Hoffmann B and Gross SR: S100A4 downregulates filopodia formation through increased dynamic instability. Cell Adhes Migr 5: 439-447, 2011. 